\title{
Modulation of fluorescence through coassembly of molecules in organic nanostructures
}

\author{
Heather A. Behanna ${ }^{1}$, Kanya Rajangam ${ }^{2}$, Samuel I. Stupp ${ }^{1,2,3,4^{*}}$ \\ ${ }^{1}$ Department of Chemistry, ${ }^{2}$ Department of Materials Science and Engineering, \\ ${ }^{3}$ Department of Materials Science \& Engineering, ${ }^{4}$ Feinberg School of Medicine, \\ Northwestern University, Evanston, IL, USA
}

Supporting Information 
a.

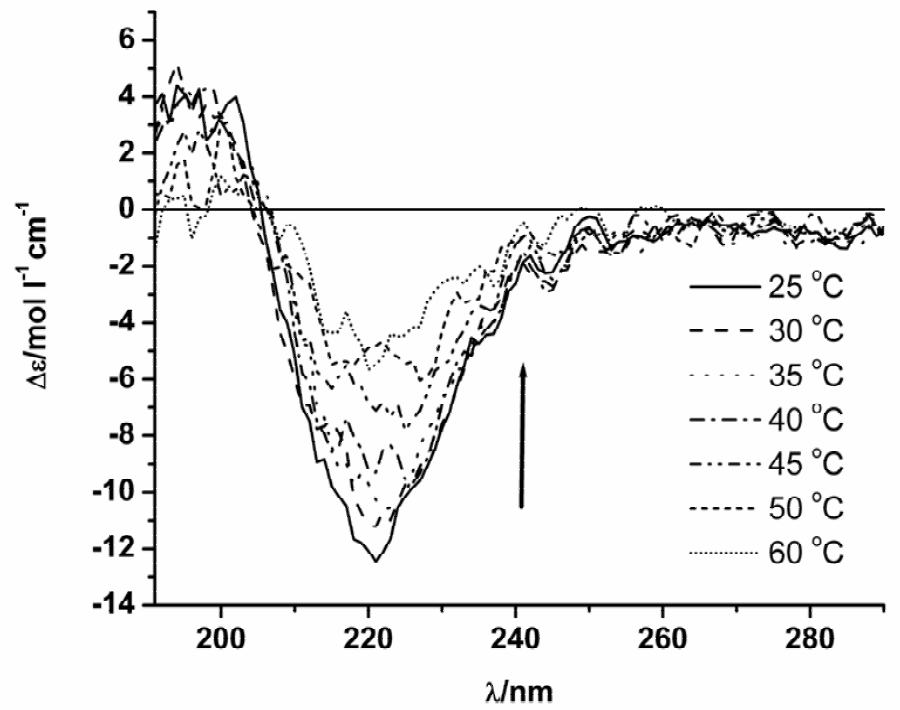

b.

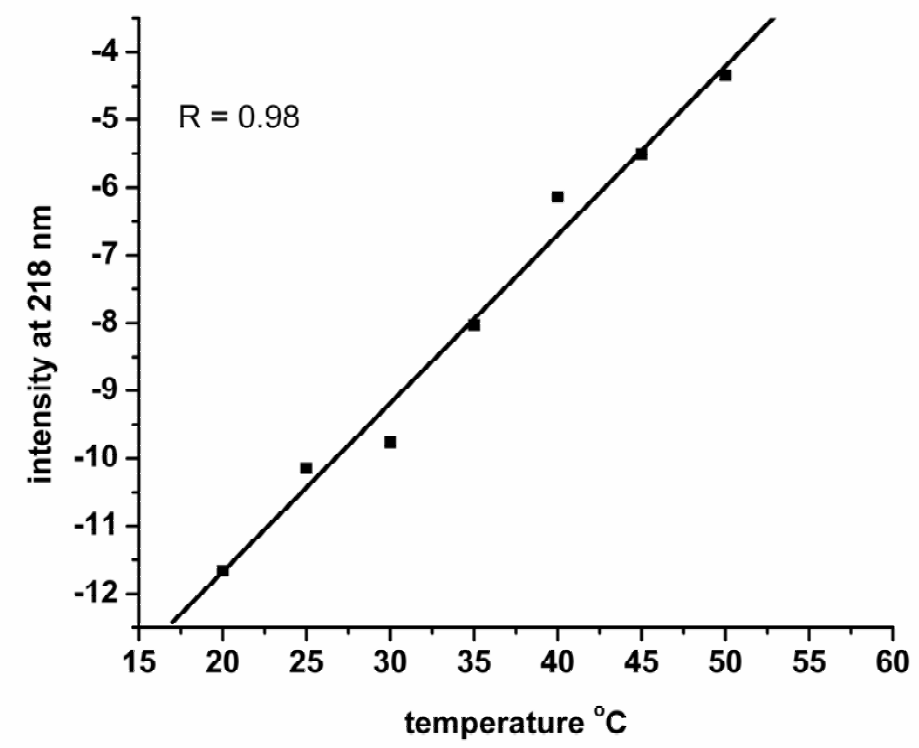

Figure 1 a) VT-CD of $\mathbf{2 / 1}$ mixed 1:1. As temperature increases from 25 to $60{ }^{\circ} \mathrm{C}$, the peak intensity decreases. This decrease is linear with temperature and is plotted in $b$ ). 

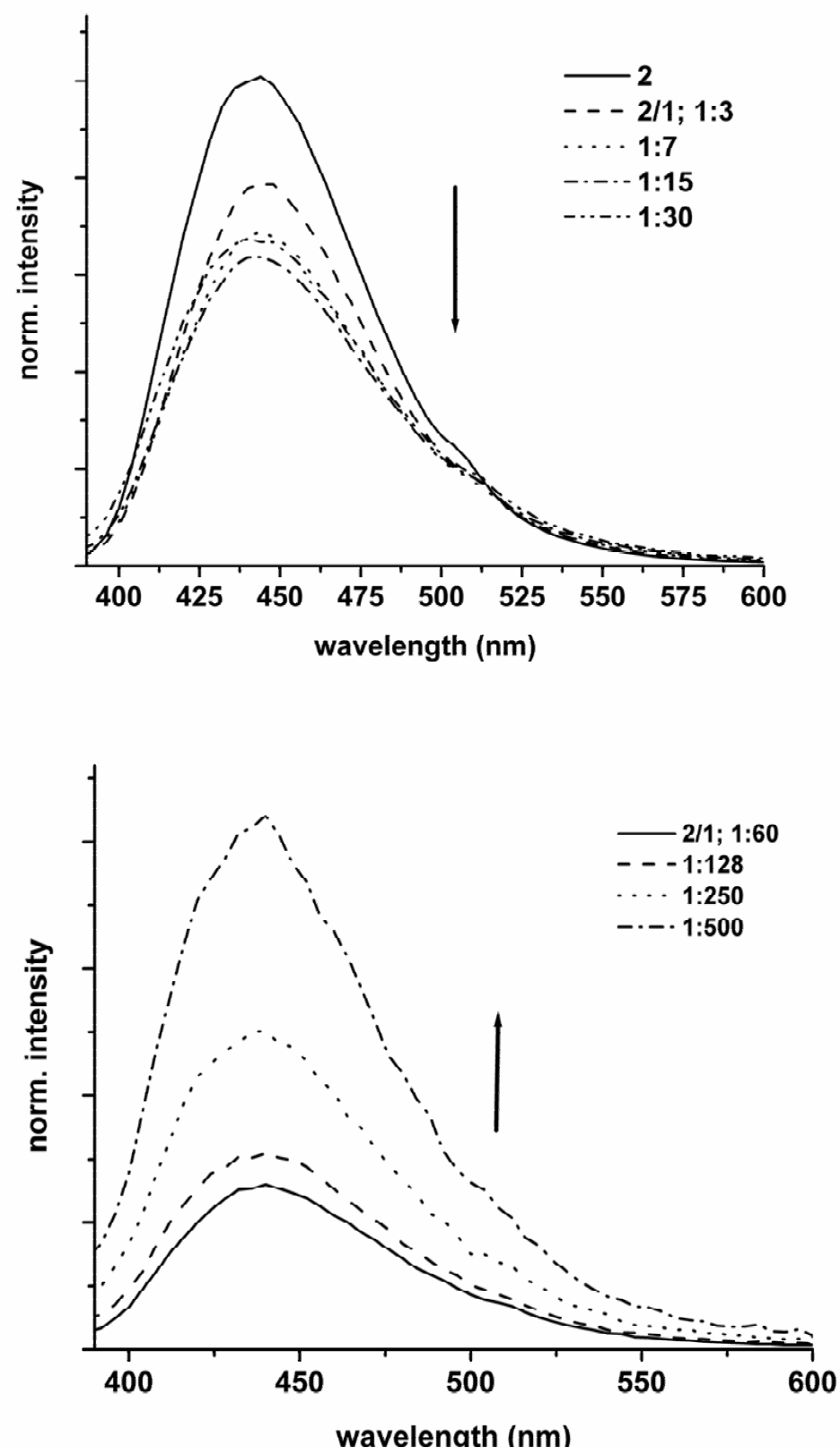

Figure2. Fluorescence of the $\mathbf{2 / 1}$ systems. a) At low dilutions, the fluorescence intensity decreases as the amount of $\mathbf{1}$ increases. b) At higher dilutions, fluorescence intensity increases as the amount of $\mathbf{1}$ increases. 

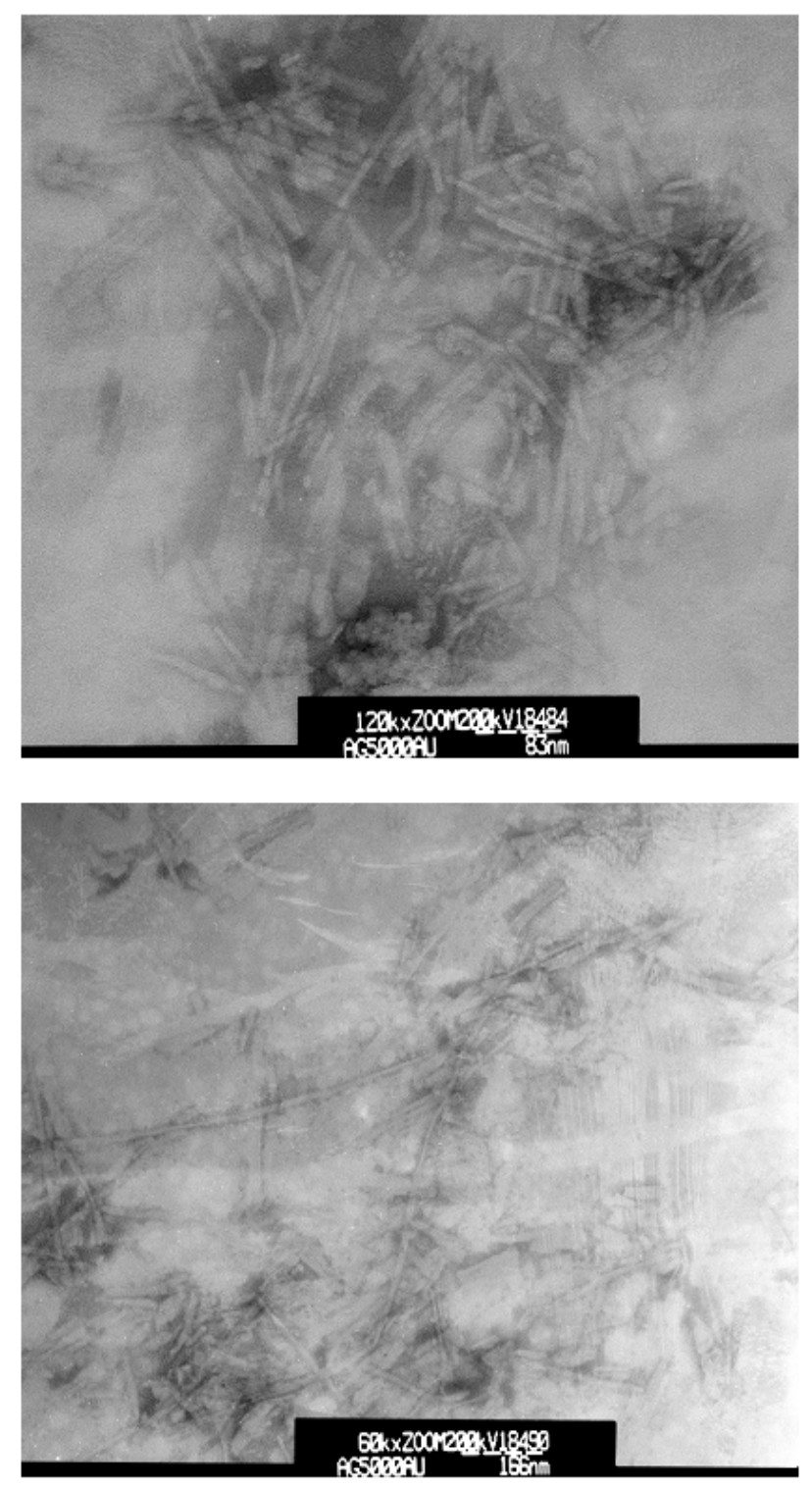

Figure 3. TEM images of $\mathbf{2 / 1}$ at 1:120 (top) and 1:500 (bottom) 


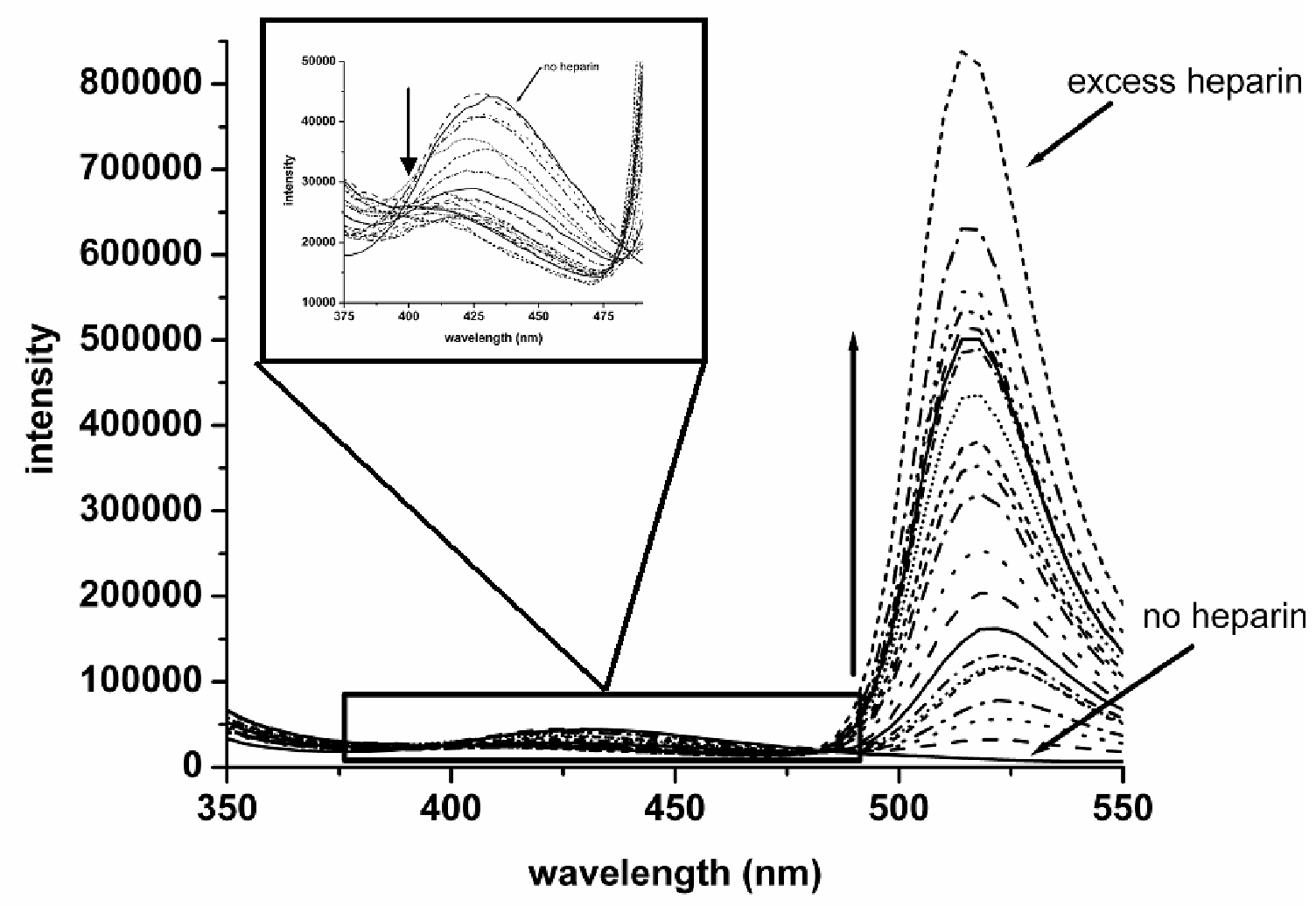

Figure 4. Fluorescence emission upon titration of 2/4 with heparin-fluorescein. Heparin was added in $5 \mathrm{uL}$ increments, and the spectra recorded after time for equilibration. The emission at $520 \mathrm{~nm}$ increased with each addition. The inset shows the emission from $\mathbf{2}$, which decreased with increasing heparin. 


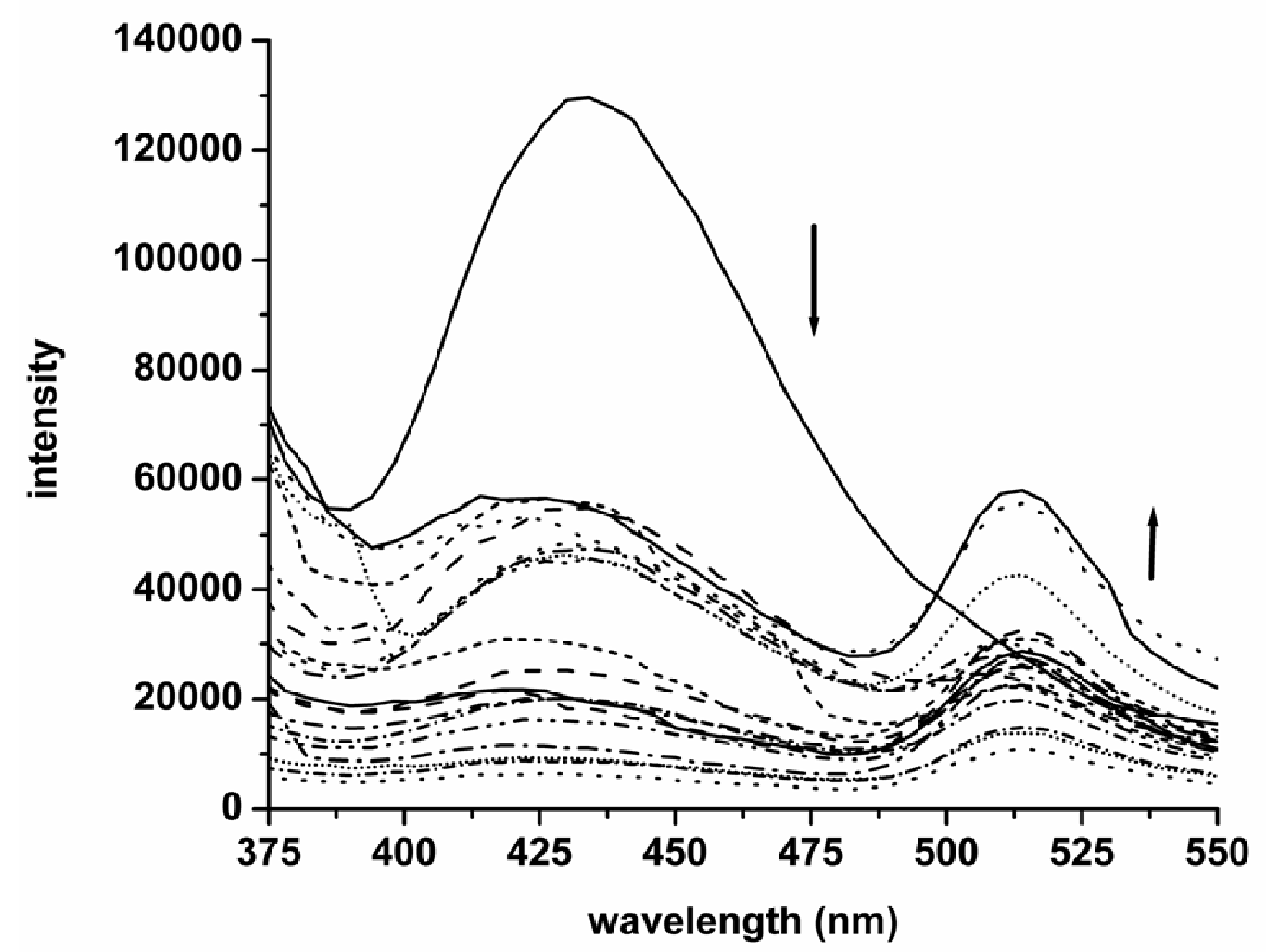

Figure 5. Titration of $\mathbf{2 / 1}$ with heparin-fluorescein. Heparin was added in $5 \mathrm{uL}$ aliquots. The emission at $520 \mathrm{~nm}$ increases slightly, and the emission of $\mathbf{2}$ decreases. 


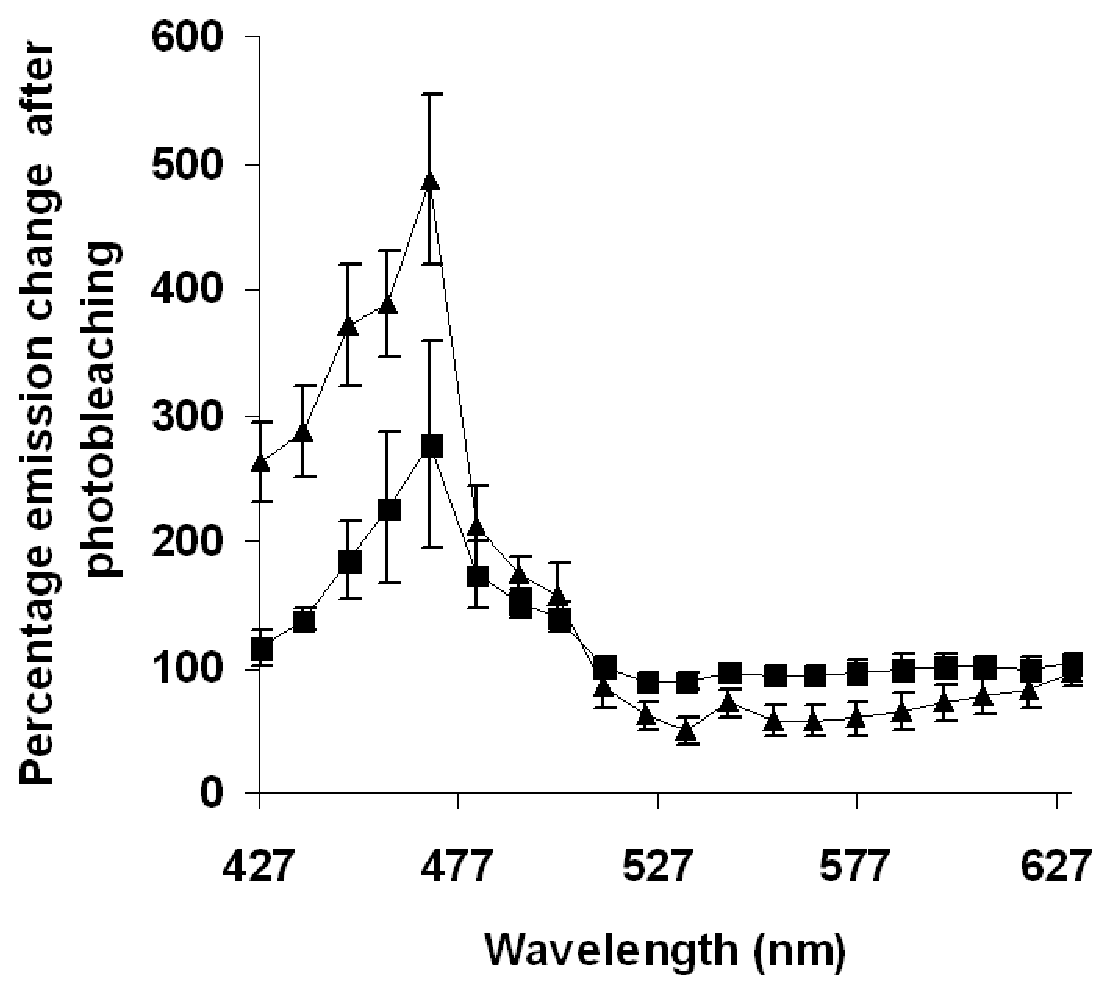

Figure 6. Graph of the ratio of fluorescence emission intensity for $\mathbf{2 / 4}$ (triangle) and $\mathbf{2 / 1}$ (square). FRET efficiency of 0.79 was obtained for $2 / 4$ versus 0.63 for the $2 / 1$ coassembly $(\mathrm{p}<0.05)$. This was calculated as previously published after normalizing for bleaching due to imaging using the formula, efficiency $=1$ - (peak prebleach donor emission / peak postbleach donor emission). In this case donor emission at $470 \mathrm{~nm}$, which was its peak emission wavelength, was used to calculate the FRET efficiency. 